\title{
DESARROLLO SOSTENIBLE DE LOS TERRITORIOS RURALES: DESAFÍOS DE LA INTERDISCIPLINARIEDAD ${ }^{1}$
}

\author{
JOÃO TORRENS \\ joaotorrens@gmail.com \\ Sociólogo e Investigador independiente \\ ORCID: 0000-0003-3907-1124
}

DOI: $10.5377 /$ rpdd.v6i1.12431

Recibido: agosto, 2021

Aceptado: septiembre, 2021

\section{RESUMEN}

T a combinación de las crisis globales y las transformaciones recientes en los Lterritorios rurales evidencian las limitaciones del paradigma dominante para explicar de forma sistémica estos cambios. Demuestran también la necesidad de nuevos referenciales para orientar la acción colectiva. Nuevos conceptos y enfoques son creados. Nuevas agendas de investigación se articulan, con base en modelos interdisciplinarios de análisis. Las bases de un paradigma emergente empiezan a ser construidas, integrando complejos campos del conocimiento. El enfoque territorial y la noción de Desarrollo Sostenible de los Territorios Rurales son parte de la transición del paradigma.

\footnotetext{
1 Este artículo presenta las principales ideas planteadas en una de las sesiones del Foro Virtual "Debate Contemporáneo sobre las Ciencias del Desarrollo Humano", particularmente en el Módulo IV, dedicado específicamente al eje temático Desarrollo Rural Sostenible. Este evento, realizado entre los días 10 y 14 de mayo de 2021, ha sido promovido por el Doctorado en Ciencias del Desarrollo Humano de la Universidad Nacional Autónoma de Honduras (UNAH) y contó con la participación de académicos e investigadores de diferentes áreas y países. La coordinación del Foro ha definido como objetivos de la referida sesión: "facilitar una mejor comprensión de la naturaleza de los nuevos paradigmas y procesos de desarrollo rural latinoamericano y aportar al debate científico sobre los grandes desafíos que enfrenta actualmente el desarrollo rural sostenible en América Latina".
} 


\begin{abstract}
The combination of global crisis and recent transformations in rural territories demonstrate the limitations of the dominant paradigm to explain these changes in a systemic way. They also reveal the need for a new framework to guide collective action. New concepts and approaches are created. New research agendas are articulated based on interdisciplinary models of analysis. The foundation of an emerging paradigm begins to be built, integrating complex fields of knowledge. The territorial approach and the notion of Sustainable Development of Rural Territories are part of the paradigm's transition.
\end{abstract}

\title{
KEYWORDS
}

Sustainable development. Rural territories. Interdisciplinarity. Paradigm.

\section{INTRODUCCIÓN}

Las posibilidades de análisis para abordar los desafíos teóricos contemporáneos sobre el Desarrollo Sostenible de los Territorios Rurales (DSTR) son muy diversas y cualquiera opción implica en el enfoque de temas dinámicos, interdependientes y complejos. Este artículo pretende analizar el DSTR a partir de la perspectiva de la interdisciplinariedad, aquí considerada como una herramienta que contribuye para el proceso de transición del paradigma dominante del conocimiento.

La adopción de la noción Desarrollo Sostenible de los Territorios Rurales ${ }^{3}$ es importante, pues integra el enfoque territorial (Berdegué, 2020) al abordaje multidimensional de la sostenibilidad (Martínez, 1997) en las reflexiones y formulaciones conceptuales sobre el desarrollo de los territorios de bases rurales. Así, esta perspectiva concibe al desarrollo territorial a partir de sus múltiples atributos (ecológico-ambientales, sociales, económicos, políticos y culturales), escalas de acción y sujetos sociales involucrados en los procesos de conformación de cada territorialidad específica.

3 En una perspectiva de análisis más amplia, se puede trabajar también con el concepto "Desarrollo Territorial Sostenible", utilizado para estudiar las dinámicas territoriales de desarrollo en zonas caracterizadas y hegemonizadas por actividades concentradas en los espacios urbanos, aunque necesariamente manténganse relaciones con los espacios rurales propiamente dichos. 


\section{DESARROLLO SOSTENIBLE DE LOS TERRITORIOS RURALES: DESAFÍOS DE LA \\ INTERDISCIPLINARIEDAD}

Por tanto, este es el contexto a partir del cual se sitúa el proceso de búsqueda de nuevos espacios de investigación y de acción colectiva, dónde en su interior emergen diversos marcos conceptuales y enfoques metodológicos que transcienden la perspectiva unidisciplinaria e implican necesariamente en una construcción interdependiente e integrada. El concepto que origina el Doctorado en Ciencias del Desarrollo Humano, la noción de Desarrollo Rural Sostenible e inúmeros conceptos que se han venido formulando en los últimos cincuenta años son parte constituyente de esta trayectoria innovadora que busca tejer nuevas conexiones para comprender la integralidad de los fenómenos investigados. Además, estos procesos de producción y gestión del conocimiento, por su carácter multidimensional e interdisciplinario, contribuyen directamente para la transición del paradigma, en la medida que facilitan la construcción de nuevos enfoques, campos de investigación y narrativas explicativas más adecuadas a las transformaciones del mundo actual.

El presente texto es fruto, por un lado, de una reflexión basada en la lectura crítica de referencias bibliográficas que analizan el significado de las investigaciones interdisciplinarias, en el marco de procesos más amplios que apuntan hacia la transición del paradigma dominante. Por otro lado, las formulaciones que serán presentadas a seguir consideran también la experiencia del trabajo de cooperación técnica realizada en diferentes países de la América Latina y el Caribe que han implementado políticas y programas de apoyo al desarrollo territorial rural. Tratase, por tanto, de un artículo que combina una reflexión teórico-cualitativa con las lecciones aprendidas de la trayectoria profesional. Así, a partir de la combinación de estos dos aprendizajes, se han elaborado cuatro ideas clave que orientarán la exposición de los argumentos de este artículo.

\section{Los desafíos de la interdisciplinariedad y la transdisciplinariedad y el cambio de paradigma}

Sin embargo, antes de pasar al análisis de los desafíos contemporáneos vinculados al tema del desarrollo sostenible de los territorios rurales, es importante desarrollar unas ideas preliminares sobre cómo las formulaciones conceptuales y las acciones colectivas que las fundamentan se articulan con el proceso más amplio de transición del paradigma del conocimiento dominante en los días de hoy. Además, se pretende demostrar que la discusión específica sobre los rumbos teóricos del DSTR mantienen estrecha relación con la construcción de nuevos enfoques, como los de interdisciplinariedad y transdisciplinariedad, comprendidos aquí como procesos indispensables al cambio de paradigma. 
El concepto de paradigma ha sido utilizado de manera muy diversa en la literatura académica y varios pensadores modernos han formulado magníficas contribuciones epistemológicas o desde la filosofía de la ciencia para explicarlo. Este artículo incorpora la noción planteada por Fritjof Capra (1992) que asocia este concepto a un conjunto de métodos e ideas que conforma una "visión de la realidad", orientando nuestra racionalidad, nuestra forma de pensar el mundo, nuestros conceptos, mentalidades, percepciones y valores. Capra advierte que el paradigma dominante ha surgido en el período del Renacimiento europeo y, desde entonces, se ha venido evolucionando y recreándose, pero, de un modo general, persisten sus premisas fundantes que "incluyen el concepto del método científico como único enfoque valido para llegar al conocimiento; la idea del universo como sistema mecánico compuesto de bloques elementales; la vida en sociedad vista como una lucha competitiva por la existencia y el crecimiento tecnológico y económico para obtener un progreso material ilimitado" (p. 16). No obstante, desde mediados del siglo pasado, diversos hallazgos provenientes de diferentes campos de la ciencia han puesto en jaque a estas concepciones y han creado las condiciones iniciales para la emergencia de un cambio de paradigma. Entre estas nuevas percepciones del mundo, como afirma Osorio, "hemos comenzado a comprender el mundo en términos de sistemas dinámicos, donde las interrelaciones entre los constituyentes de los sistemas y su entorno resultan tan importantes como el análisis de los componentes mismos. El mundo ha comenzado a dejar de ser comprendido como un conjunto de objetos para convertirse en un enjambre de conexiones, de interacciones, de redes, de emergencias, de devenires, de incertidumbres, de contradicciones" (p. 280). Así, desde esta perspectiva emergente, el mundo ya no puede más ser comprendido como una máquina que funciona mecánicamente a partir del funcionamiento de las partes, ni tampoco es posible entender la evolución de la vida sin comprender las dinámicas de los procesos de cooperación que también se establecen entre los seres vivos.

En general, los estudios sobre el tema DSTR deben ser valorizados como parte constituyente de este proceso de cambio paradigmático, pues implican en la realización de investigaciones interdisciplinarias que comparten un objeto común a partir de distintas lentes de lectura que se coordinan para ofrecer un análisis más integral. De forma complementaria, estos estudios también pueden ser percibidos como parte de una estrategia progresiva de fortalecimiento de las condiciones para la consolidación de análisis transdisciplinarios, en el marco de la conformación de campos de conocimiento más complejos.

Estas aproximaciones entre diferentes disciplinas para buscar una comprensión más integral de un proceso (como el DSTR) se presentan como una fuerte demanda 
del pensamiento moderno, pues el desarrollo técnico-científico, a partir del siglo XIX, ha producido el aparecimiento de numerosos campos disciplinarios que tienden a actuar de forma aislada entre sí. La interdisciplinariedad busca entrelazar un conjunto de disciplinas que comparten un objeto común y actúan de manera interdependiente.

En el marco de este enfoque, las "fronteras" que delimitan la especificidad de cada disciplina continúan existiendo. Las experiencias de articulación de varias disciplinas amplían las miradas de cada una, facilitando la transferencia de conocimientos de una disciplina a otra. Esto contribuye para la construcción de nuevos enfoques e, incluso, la creación de nuevos campos científicos, con sus propios métodos, instrumentos, lenguajes, objetos de estudio y análisis integrales. Pero, a pesar de la importancia de estos avances con respecto a los análisis unidisciplinarios, es preciso reconocer que la interdisciplinariedad "sigue inscrita en el marco de la investigación disciplinaria” (Nicolescu, 1996, p. 37).

La transdisciplinariedad pretende ir más allá del marco de los conocimientos disciplinares, pues, en última instancia, busca una nueva forma de organización del conocimiento, un tipo de integración más compleja de distintas áreas del saber, trascendiendo la actual estructura de los campos disciplinarios, como se conoce hoy. Permite la integración de los diferentes aportes científicos de cada disciplina y su transformación en un nuevo campo científico, organizado a partir de nuevas estructuras lógicas de disposición del conocimiento.

Entre los elementos que la distinguen de la interdisciplinariedad, se puede destacar dos aspectos fundamentales: por un lado, lanza una mirada sobre las realidades, buscando "comprender la multiplicidad de los nexos, de las relaciones y las interrelaciones que las constituyen" (Martínez, apud Osorio, 2012, p. 281). A partir del momento en que el mundo pasa a ser considerado como "un polisistema, que se reproduce y auto-reproduce a partir de, desde y por medio de la interacción de sus subsistemas, que se conciben a su vez por las relaciones entre ellos y por las relaciones entre ellos con un todo emergente (...) el conocimiento de la realidad como conocimiento de las partes y como suma de las mismas se torna profundamente inadecuado [y] hoy por hoy, además de inadecuado, ineficaz" (Osorio, 2012, p. 283). Por otro lado, el enfoque de la transdisciplinariedad postula la inexistencia de "una realidad", pues aboga en favor de la existencia de una diversidad de comprensiones de la realidad, estructuradas a partir de varios niveles de comprensión. 
Por fin, retomando las premisas de Basarab Nicolescu en el "Manifiesto de la Transdisciplinariedad", Sergio N. Osorio afirma que la principal diferencia con relación a la interdisciplinariedad es que en este abordaje el foco de la producción del conocimiento se sitúa en el conocimiento en sí mismo, mientras que la perspectiva de la transdisciplinariedad pone interés "en la dinámica de la acción de varios niveles de la realidad de cara a la comprensión del mundo presente" (2012, p. 285) y apunta para la transformación de la realidad estudiada.

Por tanto, estas consideraciones iniciales pretenden demostrar que la construcción del tema Desarrollo Sostenible de los Territorios Rurales es parte de este complejo proceso de aproximaciones interdisciplinarias que presentan un fuerte potencial no solo para confluir en la conformación de nuevos campos de investigación, pero principalmente en la constitución de campos de conocimientos muy distintos a las estructuras de las disciplinas conocidas en la actualidad.

Desafíos de la construcción teórica acerca del Desarrollo Sostenible de los Territorios Rurales

Las formulaciones teórico-metodológicas sobre el Desarrollo Sostenible de los Territorios Rurales, elaboradas en los últimos años, presentan diversos desafíos estratégicos. Para ser coherente con el enfoque del análisis elegido para este artículo, se pretende aquí formular cuatro ideas clave que enfatizan la importancia de la interdisciplinariedad como un instrumento para la transición del paradigma $y$, en particular, cómo el concepto de DSTR contribuye directamente para profundizar estos aportes.

Idea Clave n. ${ }^{\circ}$ 1. El modelo civilizatorio hegemónico en el planeta ha provocado una combinación de crisis que inciden sobre diferentes dimensiones de la vida en el planeta (ecológico-ambiental, económica, social, política y ética). Las interdependencias y sinergias producidas a partir de la interacción de estas crisis cobran de la academia análisis y respuestas integradas para superar esta crisis civilizatoria.

Desde fines del siglo XX, la humanidad ha experimentado la emergencia de un conjunto de crisis que actúan de forma interdependiente y presentan escenarios asustadores para la reproducción de la vida en el planeta, en un futuro cercano. El entrecruzamiento dinámico de estas crisis que surgen en diferentes dimensiones de las 
relaciones Naturaleza-Sociedad-Economía ${ }^{4}$, en última instancia, revela el predominio de una visión de mundo, una racionalidad, una lógica económica, principios de relacionamiento con la naturaleza y valores estructurantes de las relaciones sociales que se fundamentan en falsas premisas. Entre estos "mitos", es posible resaltar las ideas de progreso como un proceso continuo y linear, de crecimiento económico ilimitado, de libre mercado, de autorregulación del mercado, de libertad individual, de igualdad de oportunidades, de movilidad social, de la competitividad como factor determinante del éxito, del consumo como sinónimo de felicidad.

\section{Estas crisis globales se traducen en las siguientes dimensiones:}

- las crisis ecológica y ambiental que afectan el planeta y las formas de vida ocurren debido a causas humanas, y no pueden ser atribuidas a las dinámicas autorreguladoras de la Tierra, como ha ocurrido en el pasado geológico del planeta. Por la primera vez en la historia, las raíces del cambio climático están directamente relacionadas al modelo tecnológico de desarrollo económico-industrial y al modo de vida centrados en la consolidación de una matriz energética basada en el uso de combustible fósiles que provoca la consecuente emisión de gases de efectos estufa. Adicionalmente, a estos graves factores se suman el agravamiento de los procesos de deforestación, la degradación creciente de los recursos naturales, la desertificación de los suelos, la transformación de grandes áreas de forestas tropicales en sabanas, entre otros procesos que interfieren en la regulación ecológica del planeta como un todo. La conjugación explosiva de estos procesos ha impulsado serias transformaciones climáticas en el planeta (calentamiento global, crecimiento de la huella ecológica, elevación del nivel de los océanos) - factores que impactarán progresivamente en las relaciones sociales, las relaciones con la naturaleza y las actividades económicas, en varias partes del mundo;

- la crisis económica se manifiesta en la globalización desregulada, la creciente tendencia de financiarización de la economía y de sus movimientos de especulación financiera, con la emergencia del capital ficticio y del trabajo improductivo, la recesión económica prologada, la supremacía de la lógica del mercado por sobre los valores humanos y ecológicos, la reestructuración de las dinámicas del mundo del trabajo (precarización de las relaciones laborales, desempleo y empleo informal), etc.;

4 Para pensar las interrelaciones entre estas tres dimensiones, es recomendable evitar la reducción de la Economía a la idea de "Mercado". 
- la catástrofe social puede ser identificada en el crecimiento de las desigualdades y exclusiones sociales, la manutención de los altos niveles de pobreza y hambre, la elevación de los indicadores de sobrepeso y obesidad, los intensos movimientos migratorios, los efectos dramáticos del desmonte de las estructuras del Estado que restringen el acceso de la mayoría de las población a los servicios públicos, especialmente en las áreas de educación, salud, asistencia social, vivienda y saneamiento, el aumento de los procesos de individualización, las diferentes formas de violencia social - fenómenos que ocurren en diversas regiones del planeta;

- la crisis en la esfera política puede ser ejemplificada por el debilitamiento de las bases institucionales del multilateralismo en escala global, las limitaciones de los sistemas de representación política del Estado democrático basados en un diseño creado en fines del siglo XVIII, la desideologización de los partidos políticos, la amenaza permanente del conflicto nuclear, la continuidad de guerras bélicas y conflictos militares de diferentes naturalezas, la persistencia de tendencias políticas autoritarias;

- la crisis de los referenciales éticos de la sociedad se revela de diversas formas: el sentimiento de indiferencia ante los daños ecológicos y ambientales del planeta, la apatía ante las desigualdades sociales, la miseria y el hambre, las injusticias sociales, las varias formas de discriminación y violencia social (racismo, xenofobia, androcentrismo, feminicidio, prejuicio religioso o de género, el trabajo en condiciones similares a la esclavitud), la mercantilización de las relaciones sociales, la exacerbación del individualismo y consumismo, el predominio de relaciones intersubjetivas marcadas por lo efímero y fugaz, la intolerancia a las diferencias, etc. La diseminación de estos principios y valores que orientan la estructuración de las relaciones humanas y con la naturaleza evidencian la fragilización del padrón ético de la civilización.

En verdad, no existe en la realidad una separación rígida entre estas crisis, pues ellas se retroalimentan permanentemente, provocando una situación que conlleva a la identificación de una profunda crisis civilizatoria. Desde esta perspectiva multidimensional, los fundamentos civilizatorios del modelo hegemónico de organización de las sociedades se ven cuestionados debido a los impactos producidos en diferentes dimensiones de la vida. En ese sentido, las interdependencias y sinergias generadas entre estas crisis demandan de la academia y los sujetos sociales, en general, análisis holísticos y respuestas integrales que aporten para el enfrentamiento y superación de estas situaciones críticas. 


\section{DESARROLLO SOSTENIBLE DE LOS TERRITORIOS RURALES: DESAFÍOS DE LA INTERDISCIPLINARIEDAD}

Sin embargo, los países no asisten pasivamente a estos procesos de cambio. Por más que los acuerdos y compromisos firmados en el ámbito de la Organización de las Naciones Unidas aún puedan ser analizados como discursos políticos que presentan enormes dificultades para trasladarse en la práctica, por medio de la implementación de políticas públicas vinculadas a estos lineamientos globales, es preciso reconocer también que estos instrumentos internacionales representan importantes referenciales para orientar los cambios en áreas prioritarias. Entre los temas asociados al DSTR que han sido priorizados para la construcción de una agenda global pueden ser mencionados: el Pacto Internacional de Derechos Humanos Económicos, Sociales y Culturales, la Agenda 2030 para el Desarrollo Sostenible, el Acuerdo de París, el Decenio de la Agricultura Familiar.

Estas diferentes plataformas ponen en el centro de la agenda política global la relevancia de los temas ecológicos y ambientales, económicos, sociales y políticos, considerados a partir de una dinámica de interacción, interdependencia y retroalimentación continua. Además, revelan un elemento común de destaque de estos acuerdos internacionales: el reconocimiento y valorización del lugar estratégico de los territorios rurales y los diferentes actores vinculados a la agricultura para la construcción de alternativas de superación de estas crisis conjuntas que agudizan la incertidumbre y la desesperanza colectiva. Esta importancia de los procesos rurales puede ser comprobada recientemente con la crisis sanitaria provocada por la pandemia del COVID-19: por un lado, los efectos de las transformaciones rurales sobre la desregulación de los ecosistemas y el equilibrio de las condiciones ecológicas son factores determinantes para la emergencia de virus y enfermedades (Jordano, 2020); por otro lado, diversos análisis reconocen la relevancia de los espacios rurales y, en especial, la agricultura familiar para la producción de alimentos y la manutención del funcionamiento de las sociedades y las economías, en un escenario extremadamente adverso (FAO, 2021).

Idea Clave n. ${ }^{\circ}$ 2. La tendencia de emergencia y construcción de nuevos conceptos y enfoques se constituye en una respuesta a las transformaciones recientes de la ruralidad y las limitaciones del paradigma dominante para explicarlas de manera integradora y sistémica.

En las últimas décadas, las Ciencias Humanas, de una manera general, han formulado enfoques y conceptos innovadores que buscan ofrecer nuevas claves de lectura para comprender los fenómenos de la sociedad. La búsqueda por estas formulaciones conceptuales se debe a dos factores esenciales. De un lado, está asociada a la profundización y aceleración de las transformaciones de la ruralidad y la interrelación de estos cambios recientes con la complejidad de los procesos globales 
contemporáneos: (i) la construcción de sistemas alimentarios en escala global; (ii) las crecientes y diferenciadas formas de relacionamiento entre los espacios rurales y urbanos; (iii) la emergencia de nuevos actores económicos, sociales y políticos en los territorios rurales - particularmente de un sector agroindustrial vinculado a la exportación, fuertemente dependiente de los incentivos financieros del Estado y con un legado cultural preso a la grande propiedad de la tierra; (iv) el desarrollo de actividades económicas que producen profundos impactos socioambientales y aceleran los efectos de la crisis climática sobre la agricultura - expulsión de poblaciones ancestrales, migración en diferentes escalas, acaparamiento de tierras con fines de especulación financiera; (v) los conflictos entre un proyecto de desarrollo basado en el sector agroindustrial y una modalidad de desarrollo fundada en la perspectiva de las agriculturas familiares, orientada para la diversificación productiva y la agroecología, el fortalecimiento de mercados locales, la utilización de tecnologías de menor impacto ambiental, la adopción de prácticas resilientes al cambio climático, etc. De esta manera, los cambios actuales en los territorios rurales de la América Latina y el Caribe imponen la necesidad de formular marcos conceptuales de referencia de características interdisciplinarias. Con base en estas nuevas referencias se pretende analizar estas transformaciones con mayor rigor científico y, al mismo tiempo, brindar análisis para que los actores sociales dispongan de nuevos instrumentos que les permitan desarrollar estrategias de acción distintas del pasado.

El segundo aspecto que justifica la formulación de nuevos enfoques son las limitaciones explicativas del paradigma dominante, caracterizado por el predominio de análisis centradas en visiones sectoriales, fragmentadas, compartimentadas, simplificadoras y reductoras de la complejidad de las ruralidades. Los enfoques dominantes tienden a analizar a un determinado fenómeno desde una perspectiva unidisciplinaria, basada en la visión cartesiana aún dominante que establece la necesidad de comprender, de forma segmentada, las partes del "objeto de estudio". Desde esta concepción, la comprensión del todo deriva de la suma de las partes. Sin embargo, los aspectos estructurales de los territorios rurales en el mundo contemporáneo - y mismo los del pasado - no pueden ser reducidos a piezas de un reloj, como se fuesen determinados por leyes mecánicas. Esta concepción, como ha enseñado Nicolescu, además de inadecuada, es insuficiente para explicar las actuales transformaciones.

En ese sentido, los procesos de producción y gestión del conocimiento impulsados por estudiosos que incorporan estas nuevas herramientas buscan: (i) establecer una nueva agenda de investigación para las ciencias vinculadas al DSTR; (ii) crear nuevas lógicas, racionalidades y narrativas explicativas de los fenómenos actuales, enfatizándose la multidimensionalidad, la diversidad y la interdependencia; 
(iii) formular análisis sistémicos y explicaciones holísticas acerca de las ruralidades. Estos estudios abren espacio para consolidar el enfoque interdisciplinario y, al mismo tiempo, tienen el potencial de construir nuevos campos de conocimiento que se encuentran más allá de cada disciplina cooperante. Los problemas globales y locales de los territorios rurales no se prestan para ser analizados de manera aislada por una racionalidad lógica estructurada como bloques de disciplinas que generan pocos espacios de interacción interdisciplinaria.

Idea Clave n. ${ }^{\circ}$ 3. Los debates contemporáneos sobre la construcción de un Desarrollo Sostenible de los Territorios Rurales se sitúan, por tanto, en el marco de la emergencia de nuevos conceptos y enfoques, agendas de investigación interdisciplinaria y plataformas innovadoras de acción colectiva (redes y articulaciones), que contribuyen para la construcción de un nuevo paradigma.

Los avances en las construcciones teóricas, alcanzados por medio de la cooperación entre diferentes disciplinas, en verdad, reflejan el fortalecimiento de los procesos de acción colectiva y de conformación de plataformas políticas (articulaciones, redes y movimientos sociales) que buscan incidir en los diversos espacios de decisión con la finalidad de contestar los efectos de las estrategias de desarrollo global. Es a partir de este contexto que las fuerzas de la sociedad civil y la academia se movilizan para formular nuevos enfoques y conceptos, pues el cambio de paradigma no es una acción que se produce solamente en el ámbito de las ideas, de las ciencias y del conocimiento. En el caso de las ciencias humanas, económicas o ambientales, el "laboratorio" para la construcción del conocimiento es la dinámica cambiante de las realidades, siempre permeadas por relaciones de poder político que imponen obstáculos y resistencias a los cambios. Este es el substrato principal de las reflexiones intelectuales y, justamente debido a estas circunstancias, la transición del paradigma no se hace en pocas décadas de elaboración-acción. Este cambio es parte de un proceso de transformación histórica de las sociedades, con sus avances y retrocesos políticos y sociales.

Por eso mismo, es preciso valorizar la importancia de los enfoques de derechos, sostenibilidad, resiliencia, equidad, género, territorial, empoderamiento, entre otros, elaborados desde fines del siglo XX. Estos aportes demuestran la capacidad creativa del conocimiento para formular nuevas herramientas que alimentan el cambio del paradigma hegemónico. Sin embargo, los desafíos actuales impuestos por las transformaciones recientes y por el propio tiempo requieren nuevas elaboraciones, pues los análisis futuros sobre el Desarrollo Sostenible de los Territorios Rurales ya no deben limitarse a reproducir mecánicamente las premisas epistemológicas de 
los conceptos y enfoques construidos en las últimas décadas. Es preciso revisarlos, renovarlos y actualizarlos permanentemente. Por tanto, es imprescindible en este momento histórico avanzar en la construcción de análisis que articulen distintos enfoques, que integren diferentes dimensiones y que aborden la complejidad de las interacciones entre Estado-Sociedad-Economía (instituciones-actores socialessectores económicos), en las diversas escalas de intervención vinculadas al DSTR.

Las investigaciones sobre este tema tan amplio permiten la integración de estos diferentes enfoques (o parte de ellos) como un ejercicio de construcción de nuevos campos de análisis interdisciplinarios, capaces de articular temas específicos y formular una visión sistémica sobre cómo los procesos ecológicos, sociales, económicos, políticos, culturales y éticos interactúan entre sí en los espacios rurales. La combinación de enfoques complementarios y convergentes facilitan la configuración y consolidación de nuevos campos de investigación, como las nociones de Sistemas Alimentarios Sostenibles, de multifuncionalidad de la ruralidad y de Sistemas Territoriales 5 . Con el objetivo de detallar este ejercicio, se puede tomar el concepto de Sistemas Alimentarios para identificar sus múltiples implicaciones (FAO, 2017).

Esta categoría supone una lógica de estructuración de los encadenamientos productivos y económicos para la producción, transformación y consumo de los alimentos, una compleja arquitectura de instituciones, políticas públicas, centros de investigación e innovación, un conjunto de infraestructuras de logística, transporte y comercialización, relaciones entre productores y consumidores, capacidades organizativas de los productores rurales, impactos de estos procesos en la crisis climática y la preservación de la biodiversidad y los saberes locales, efectos de la alimentación en la salud humana y las tradiciones culturales vinculadas a la gastronomía, cambio de los regímenes alimentarios locales con la introducción de alimentos que no son considerados como "comida de verdad". Desde esta perspectiva, la definición de Sistemas Alimentarios implica en una visión multidimensional e integradora de los procesos vinculadas a la alimentación.

5 El concepto de multifuncionalidad de la ruralidad implica también en una concepción sistémica sobre las distintas contribuciones de los espacios rurales y sus actores para el "buen vivir" de una sociedad. Entre estas contribuciones, es importante rescatar los servicios ecosistémicos, la realización de actividades rurales noagrícolas, la implementación de actividades vinculadas a la bioeconomía, la producción de energías renovables, la preservación de distintos modos de vida y formas de manifestación cultural. Por otro lado, el concepto de Sistemas Territoriales presenta el potencial de analizar los territorios rurales desde la perspectiva innovadora de la Teoría de los Sistemas, como una noción que implica necesariamente en la interacción de diferentes dimensiones y escalas de acción (Samper, 2016). 


\section{DESARROLLO SOSTENIBLE DE LOS TERRITORIOS RURALES: DESAFÍOS DE LA INTERDISCIPLINARIEDAD}

En ese sentido, este conjunto innovador de conceptos y enfoques ofrece nuevas lentes de lectura y contribuye para establecer las condiciones para la construcción de nuevas lógicas explicativas, nuevos lenguajes, nuevas narrativas explicativas, o sea, de una mirada de carácter integrador, sistémico y multidimensional. Del punto de vista de la transición del paradigma, un paso esencial es la necesidad de entrelazar conocimientos dispersos, promoviendo las conexiones conceptuales y metodológicas entre estos abordajes, con el propósito de construir una formulación más integrada acerca de las transformaciones del mundo contemporáneo. Desde este enfoque, los centros de investigación implicados en la construcción de una agenda académica interdisciplinaria tienen la oportunidad y el desafío de construir nuevos procesos de convergencia de conocimientos, privilegiando investigaciones y estudios de recorte interdisciplinario.

Idea Clave n. ${ }^{\circ}$ 4. La diversidad de los temas imbricados en el DSTR ofrece condiciones favorables para tender puentes multidisciplinarios con potencialidades para moldear nuevos campos de investigación.

La construcción de estas convergencias de enfoques y temáticas tiene por finalidad crear nuevos puentes interdisciplinarios, nuevas mediaciones disciplinarias, superando la perspectiva de estudios basada en una simple yuxtaposición de conocimientos y aportando herramientas para la transición paradigmática. Además, otro desafío estructural del conocimiento en el área del DSTR es articular los análisis de los "temas específicos" desde una perspectiva multidimensional, identificándose cómo en un país o un determinado territorio se integran los procesos ecológicos, sociales, económicos, culturales y políticos, y cuáles son sus dinámicas de interacción y complementariedad. El desarrollo de este ejercicio académico implica en una articulación coherente de miradas y abordajes.

Sin embargo, es importante resaltar que la apertura de estos nuevos campos interdisciplinarios no invalida o niega el valor de los análisis de temas sectoriales, que necesitan ser profundizados y renovados. Las investigaciones vinculadas a "temas específicos" (como dinamización económica rural-urbana, reconfiguración de los sistemas de producción, preservación del patrimonio biocultural, estrategias de gobernanza climática, reducción de las desigualdades sociales, construcción de nuevas relaciones de género en las zonas rurales, entre otros) continuarán importantes para la comprensión de estos fenómenos. No se pretende aquí rebajar la relevancia de estos análisis, ni tampoco crear una falsa dicotomía entre los enfoques disciplinarios e interdisciplinarios. 
Por fin, estos enfoques, conceptos y campos de interdisciplinarios de investigación académica serán fundamentales para comprender, a partir de un enfoque sistémico, el significado y alcance de las profundas transformaciones en curso en los territorios rurales y en sus relaciones dinámicas con los procesos más amplios de desarrollo de las sociedades, en escala nacional, regional y global. Al mismo tiempo, estos análisis reúnen también el potencial para apoyar el diseño de nuevas relaciones sociales, nuevas relaciones entre Estado-Sociedad-Economía y relaciones entre Sociedad-Naturaleza más apropiadas para la reproducción de la vida en el planeta en el siglo XXI.

\section{CONCLUSIONES}

La diversidad y riqueza de los estudios contemporáneos sobre los temas vinculados al "desarrollo rural" revelan que la producción del conocimiento en esta área sigue una trayectoria dinámica e innovadora, mismo considerando la tendencia global de la urbanización. Los análisis alineados con la perspectiva del desarrollo rural sostenible o del desarrollo sostenible de los territorios rurales, así como otras líneas de investigación enfocadas en abordajes complementarios, de un modo general, demuestran la necesidad de producir conocimientos que respondan analíticamente a las complejas transformaciones desarrolladas en los espacios rurales en las últimas décadas. Por eso, es cada vez más presente la búsqueda para establecer aproximaciones y convergencias interdisciplinarias e intertemáticas, con la finalidad de conformar nuevos campos de investigación, con lógicas racionales y lenguajes distintos de aquellos existentes actualmente.

No se trata de "ensamblar conocimientos dispersos", pues la gestión inter o transdisciplinaria de conocimientos no funciona como piezas de un engranaje que se encajan mecánicamente entre sí para, juntas, formar un determinado objeto. Este proceso es distinto de una yuxtaposición de conocimientos que se suman para fornecer una visión "ensamblada" de los "componentes" del fenómeno analizado. Más que un "bricolaje", considerado como la unión mecánica de las partes aisladas de un todo y que no poseen espacios de interacción orgánica entre sí, la interdisciplinariedad evoca a la capacidad de entretejer saberes y conocimientos originarios de diferentes áreas, con el propósito de crear nuevas racionalidades y narrativas explicativas. En ese sentido, la interconexión de conocimientos supone la interdependencia y la producción de sinergias entre campos distintos de las ciencias, buscando formular ideas y análisis innovadoras, complementarias e integradoras, cuyas conclusiones difícilmente serían elaboradas con una visión unidisciplinaria. 


\section{DESARROLLO SOSTENIBLE DE LOS TERRITORIOS RURALES: DESAFÍOS DE LA INTERDISCIPLINARIEDAD}

Esta concepción de producción y gestión del conocimiento aún encuentra resistencias en las instituciones académicas, reguladas por los principios del paradigma dominante en las Ciencias Sociales que prevén la necesidad de descomponer el todo en sus partes, compartimentando el conocimiento, de un modo general, en disciplinas aisladas una de las otras. Sin embargo, las tendencias emergentes en diversas áreas de las ciencias, en especial en las Ciencias Humanas, demandan la construcción de nuevas conexiones científicas y campos interdisciplinarios de investigación capaces de fundar comprensiones más holísticas de las realidades. Los actuales desafíos teóricos del campo de análisis vinculado al DSTR apuntan justamente en esta dirección.

\section{BIBLIOGRAFÍA}

Berdegué, J. et al (eds.) (2020). Quince Años de Desarrollo Territorial Rural en América Latina y el Caribe: ¿Qué nos muestra la experiencia? Buenos Aires. Teseo. 324 p. Disponible en: https://www.editorialteseo.com/archivos/17437/quince-anosde-desarrollo-territorial-rural-en-america-latina/.

FAO (2017). Reflexiones sobre el Sistema Alimentario y perspectivas para alcanzar su sostenibilidad en América Latina y el Caribe. Santiago. FAO. 20 p. Disponible en: http://www.fao.org/3/a-i7053s.pdf.

FAO (2021). La transparencia y la apertura de los mercados de productos alimenticios es vital para la seguridad alimentaria y nutricional. Roma. FAO. 10 de marzo de 2021. Disponible en: http://www.fao.org/news/story/es/item/1380278/icode/.

Jordano, Pedro (2020). "Detrás de esta pandemia está la acción humana sobre la naturaleza". Entrevistado por Javier López Rejas. Revista Electrónica El Cultural España. 25.03.2020. Disponible en: https://elcultural.com/pedro-jordano-detras-deesta-pandemia-esta-la-accion-humana-sobre-la-naturaleza.

Martínez, Luciano (1997). Hacia una visión multidimensional del desarrollo sostenible en el medio rural: aproximación al caso de comunidades indígenas de la sierra central. In: Martínez, L. (comp.). El Desarrollo Sostenible en el Medio Rural. Quito. FLACSO. p. 41-59. Disponible en: https://biblio.flacsoandes.edu.ec/shared/ biblio_view.php?bibid $=5835 \&$ tab $=$ opac.

Nicolescu, Basarab (1996). La Transdisciplinariedad. Manifiesto. Hermosillo. Ed. 7 Saberes. Multiversidad Mundo Real Edgar Morin. 104 p. Disponible en: http:// basarab-nicolescu.fr/BOOKS/Manifeste_Espagnol_Mexique.pdf. 
Samper, Mario (2016). Sistemas Territoriales de Agricultura Familiar. IICASIGET: Fascículos conceptuales, n. ${ }^{\circ}$ 1. San José. IICA. 52 p. Disponible en: $\quad$ https://repositorio.iica.int/bitstream/handle/11324/6943/BVE18040112e. pdf? sequence $=1$ \&isAllowed $=\mathrm{y}$.

\section{AGRADECIMIENTOS}

Agradezco a la Profesora Dra. Margarita Oseguera de Ochoa, Coordinadora del Doctorado en Ciencias del Desarrollo Humano de la Universidad Nacional Autónoma de Honduras (UNAH), y al Profesor Doctor Mario Samper Kutschbach, también vinculado a este Doctorado, por el incentivo para elaborar y publicar estas reflexiones personales en la Revista Perspectivas del Desarrollo. 